\title{
Reinventing the displacement left ventricular assist device in the continuous-flow era: TORVAD, the first toroidal-flow left ventricular assist device
}

\author{
Carlo R. Bartoli ${ }^{1}$, Jeffrey R. Gohean ${ }^{2}$, Richard W. Smalling ${ }^{2,3}$ \\ ${ }^{1}$ Division of Cardiovascular Surgery, Hospital of the University of Pennsylvania, Philadelphia, PA, USA; ${ }^{2}$ Windmill Cardiovascular Systems, Inc., \\ Austin, TX, USA; ${ }^{3}$ Division of Cardiology, Department of Internal Medicine, University of Texas Health/McGovern Medical School, Houston, \\ TX, USA \\ Correspondence to: Carlo R. Bartoli, MD, PhD. Hospital of the University of Pennsylvania, Division of Cardiovascular Surgery, 380 South University \\ Ave, Hill Pavilion, Room 410B, Philadelphia, PA 19104, USA. Email: carlo.bartoli@uphs.upenn.edu.
}

Submitted Sep 29, 2020. Accepted for publication Feb 01, 2021.

doi: 10.21037/acs-2020-cfmcs-19

View this article at: http://dx.doi.org/10.21037/acs-2020-cfmcs-19

\section{Introduction}

Over the past two decades, left ventricular assist device (LVAD) support has emerged as standard therapy for adult patients with advanced, life-threatening heart failure. Firstgeneration pulsatile devices have been replaced by rotary blood pumps that continuously unload the failing left ventricle. So-called continuous-flow LVADs (CF-LVADs) are smaller, more reliable, durable, and energy efficient, and less traumatic to implant than first-generation devices. Survival of $80 \%$ at one year and $70 \%$ at two years has been widely achieved (1). However, CF-LVADs have introduced a new, non-pulsatile physiology in which blood encounters supraphysiologic shear stress, and as a result, unforeseen consequences have emerged.

CF-LVADs operate with an impeller that spins at thousands of revolutions per minute (RPM) to generate forward flow. Within current-generation devices, blood passes through narrow flow gaps (50 to $500 \mu \mathrm{m})$ at high velocity. Consequently, blood encounters shear stress that exceeds physiologic values by more than two orders of magnitude. Indeed, current-generation LVADs generate peak shear stress of up to $1,500 \mathrm{~Pa}$ (2). For reference, physiologic levels of intravascular shear stress in mammals are approximately two to eight $\mathrm{Pa}$ (2).

Irrefutable evidence demonstrates that supraphysiologic shear stress from CF-LVADs causes blood trauma that contributes to adverse events. Pathologic degradation of von Willebrand factor (VWF) leads to an acquired bleeding diathesis, gastrointestinal angiodysplasia, and gastrointestinal bleeding in $20 \%$ to $40 \%$ of LVAD patients $(3,4)$. Platelet activation and (at least subclinical) hemolysis cause a procoagulant state that contributes to LVAD thrombosis and thromboembolism (5). As a result, "LVAD hemocompatibility", a term that characterizes the clinical impact of biophysical interactions and blood trauma at the blood-device interface, is gaining attention as a major area for improvement of future-generation LVADs.

Lack of pulsatile blood flow has also raised concerns. Potential benefits of pulsatility include prevention of aortic valve thrombosis, leaflet fusion, de novo aortic insufficiency, favorable myocardial remodeling, avoidance of arterial stiffening, better end-organ function, reduced bleeding, and reduced stroke (6). Consequently, pulsation algorithms are being developed to generate pulsatility with CF-LVADs.

Also problematic, CF-LVADs operate at fixed RPM, independent of patient physiology. These devices do not sense or adjust device parameters in response to dynamic changes in cardiac rate, rhythm, preload, afterload, intracardiac hemodynamics, or systemic metabolic demands. "Physiologic control", a term used to describe automatic responsiveness to changes in patient physiology, is another focus for improvement of future-generation LVADs. Devices with physiologic control may sense and adjust to pathologic conditions such as exacerbation of heart failure, hypotension, hypovolemia, and malignant arrhythmia. Optimization of ventricular (un)loading may increase 

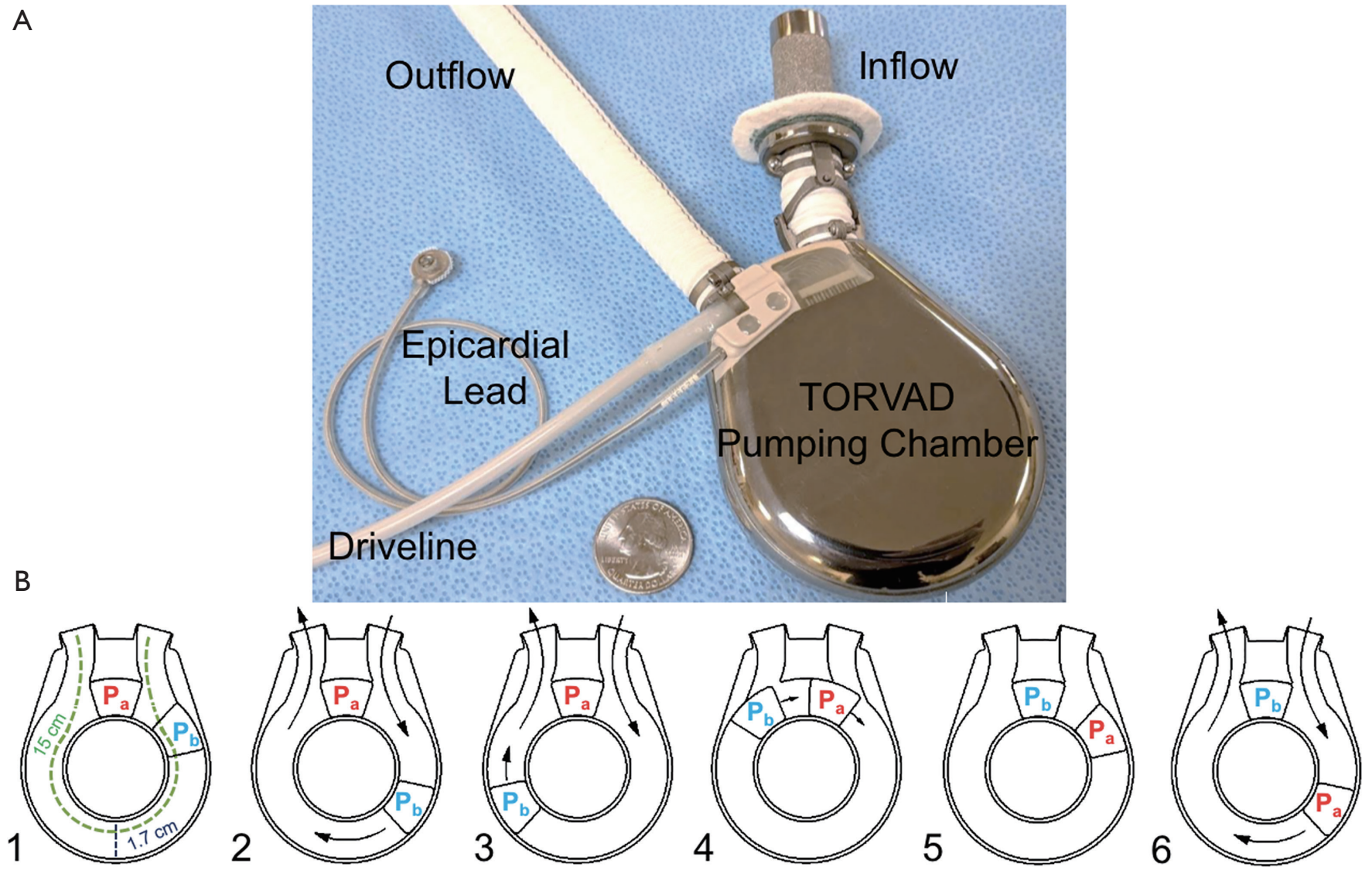

Figure 1 Toroidal-Flow TORVAD, Device and Mechanism of Flow. (A) The toroidal-flow TORVAD consists of an inflow cannula with sewing ring, torus pumping chamber, and outflow graft. An epicardial ECG lead senses the patient's native heart rhythm and triggers TORVAD support with asynchronous or synchronous pulsatile, counter-pulsatile, or co-pulsatile pumping modes. (B) To simultaneously fill and eject, the TORVAD spins two magnetic pistons ( $\mathrm{Pa}$ and $\mathrm{Pb}$ ) in sequence within the doughnut-shaped torus chamber. During support, each piston remains stationary while the other piston spins. While the first piston $(\mathrm{Pa})$ is temporarily fixed as a virtual valve between the torus inflow and outflow, the second piston $(\mathrm{Pb})$ rotates within the torus to eject $30 \mathrm{~mL}$ of blood. After one cycle, the pistons switch positions, and the first piston $(\mathrm{Pa})$ spins while the second piston $(\mathrm{Pb})$ remains stationary. The result is unidirectional, pulsatile blood flow with low shear stress and a high level of physiologic control. ECG, electrocardiogram.

favorable myocardial remodeling and facilitate LVAD weaning and explantation in the setting of myocardial recovery.

Finally, full sternotomy and cardiopulmonary bypass (CPB) deter referral of less sick patients for LVAD therapy. Minimally-invasive implantation strategies without CPB that include mini-thoracotomy, subxiphoid access, device placement in an infraclavicular pacemaker-like pocket, and percutaneous implantation may increase referral of earlierstage heart failure patients and increase the public health impact of LVAD support.

In summary, next-generation LVADs are needed to reduce adverse events and improve outcomes. Toroidal- flow is a novel LVAD flow strategy designed to improve hemocompatibility while providing pulsatility with physiologic device control. As such, the first-of-its-kind, toroidal-flow TORVAD is a disruptive technology with the potential to alter mechanical circulatory support therapy.

\section{TORVAD}

Windmill Cardiovascular Systems, Inc. (Austin, TX, USA) is developing the TORVAD (2,7-10), a positivedisplacement, rotary LVAD (Figure 1). The toroidal-flow mechanism generates low shear stress (7), minimal blood trauma (2), pulsatile blood flow (9), and operates with 
physiologic control $(8,10)$.

The pumping mechanism of the TORVAD is distinctly different from first-generation, membranetype, pulsatile LVADs and second-generation, impellerbased CF-LVADs. To simultaneously fill and eject, the TORVAD spins one of two magnetic pistons within a torus (doughnut-shaped) chamber. While the first piston is temporarily fixed as a virtual valve between the torus inflow and outflow, the second piston spins within the torus to generate unidirectional, pulsatile blood flow (Figure $1 \mathrm{~B}$ ). At $1.7 \mathrm{~cm}$, the major blood flow path is comparable to a large artery. At 35 to $250 \mathrm{RPM}$, the $30 \mathrm{~mL}$ stroke volume generates approximately one to eight liters per minute of blood flow with peak shear stress of approximately $10 \mathrm{~Pa}(9)$.

Low shear stress with the TORVAD causes minimal blood trauma. In ex vivo mock circulatory loop experiments with human blood, the TORVAD caused no platelet activation, no hemolysis, and minimal VWF degradation (2). Similarly, over 60 days, sheep implanted with a TORVAD did not demonstrate significant hemolysis or VWF degradation. The experimental animals were not anticoagulated and thromboembolism was not observed. These results suggest that excellent hemocompatibility with the TORVAD will reduce blood trauma-related adverse events.

The TORVAD operates with physiologic control. The pumping algorithm, triggered by the patient's native electrocardiogram, accommodates a variety of (patho)physiologic conditions. Dynamic changes in preload and afterload are sensed as forces against the magnetic pistons and trigger appropriate changes in piston cycling to automatically adjust hemodynamic support $(8,9)$. Systemic vascular resistance is estimated to assist in patient monitoring (10). Preload sensitivity prevents potentially dangerous suction events and overpumping (8). Asynchronous pulsatile and synchronous co-pulsatile and counter-pulsatile modes may optimize ventricular (un)loading to facilitate favorable myocardial remodeling and device weaning.

Recent large animal implants and cadaver-fit studies demonstrated that the TORVAD may be implanted via a mini-thoracotomy without CPB. Percutaneous trans-septal inflow cannula placement with implantation of the device in an infraclavicular pacemaker-like pocket is also under development.

Finally, the TORVAD design is scalable for application in pediatric patients (7). A smaller TORVAD may provide an urgently needed alternative option for pediatric mechanical circulatory support in a variety of pediatric patients with four-chambered or single ventricle physiology.

\section{Conclusions}

Device-related complications with current-generation LVADs limit widespread application in only the sickest heart failure patient. Next-generation devices with better outcomes are necessary before LVAD support is more widely adopted. Devices designed with lower shear stress will improve hemocompatibility and reduce blood trauma-related adverse events. Pulsatility and physiologic device control are needed to recreate native physiology, improve device performance, promote myocardial recovery, and facilitate LVAD weaning toward explantation. Less-invasive surgical approaches may increase patient referral for LVAD support.

Toroidal-flow is a novel flow strategy designed to improve hemocompatibility, provide pulsatility, and operate with physiologic control. As such, the toroidal-flow TORVAD is a disruptive technology with the potential to alter mechanical circulatory support therapy.

\section{Acknowledgments}

Funding: None.

\section{Footnote}

Conflicts of Interest: CRB - Windmill Advisory Board; JRG - Windmill Employee, Director of Engineering; Partial Ownership; RWS - Windmill Employee, CEO; Partial Ownership.

Open Access Statement: This is an Open Access article distributed in accordance with the Creative Commons Attribution-NonCommercial-NoDerivs 4.0 International License (CC BY-NC-ND 4.0), which permits the noncommercial replication and distribution of the article with the strict proviso that no changes or edits are made and the original work is properly cited (including links to both the formal publication through the relevant DOI and the license). See: https://creativecommons.org/licenses/by-nc-nd/4.0/.

\section{References}

1. Kirklin JK, Pagani FD, Kormos RL, et al. Eighth annual INTERMACS report: Special focus on framing the impact of adverse events. J Heart Lung Transplant 
2017;36:1080-6.

2. Bartoli CR, Hennessy-Strahs S, Gohean J, et al. A Novel Toroidal-Flow Left Ventricular Assist Device Minimizes Blood Trauma: Implications of Improved Ventricular Assist Device Hemocompatibility. Ann Thorac Surg 2019;107:1761-7.

3. Bartoli CR, Restle DJ, Zhang DM, et al. Pathologic von Willebrand factor degradation with a left ventricular assist device occurs via two distinct mechanisms: mechanical demolition and enzymatic cleavage. J Thorac Cardiovasc Surg 2015;149:281-9.

4. Bartoli CR, Zhang DM, Hennessy-Strahs S, et al. Clinical and In Vitro Evidence That Left Ventricular Assist Device-Induced von Willebrand Factor Degradation Alters Angiogenesis. Circ Heart Fail 2018;11:e004638.

5. Bartoli CR, Zhang D, Kang J, et al. Clinical and In Vitro Evidence That Subclinical Hemolysis Contributes to LVAD Thrombosis. Ann Thorac Surg 2018;105:807-14.

6. Bartoli CR, Atluri P. Do patients with a continuousflow left ventricular assist device benefit from induced-

Cite this article as: Bartoli CR, Gohean JR, Smalling RW. Reinventing the displacement left ventricular assist device in the continuous-flow era: TORVAD, the first toroidalflow left ventricular assist device. Ann Cardiothorac Surg 2021;10(2):274-277. doi:10.21037/acs-2020-cfmcs-19 pulsatility or are we just spinning our wheels? J Thorac Cardiovasc Surg 2015;150:945-6.

7. Gohean JR, Larson ER, Hsi BH, et al. Scaling the LowShear Pulsatile TORVAD for Pediatric Heart Failure. ASAIO J 2017;63:198-206.

8. Gohean JR, Larson ER, Longoria RG, et al. Preload Sensitivity with TORVAD Counterpulse Support Prevents Suction and Overpumping. Cardiovasc Eng Technol 2019;10:520-30.

9. Letsou GV, Pate TD, Gohean JR, et al. Improved left ventricular unloading and circulatory support with synchronized pulsatile left ventricular assistance compared with continuous-flow left ventricular assistance in an acute porcine left ventricular failure model. J Thorac Cardiovasc Surg 2010;140:1181-8.

10. Rapp ES, Pawar SR, Gohean JR, et al. Estimation of Systemic Vascular Resistance using Built-In Sensing From an Implanted Left Ventricular Assist Device. J Eng Sci Med Diagn Ther 2019;2:041008. 